\title{
Surflets: A Sparse Representation for Multidimensional Functions Containing Smooth Discontinuities
}

\author{
Venkat Chandrasekaran, Michael B. Wakin, Dror Baron, and Richard G. Baraniuk ${ }^{1}$ \\ Department of Electrical and Computer Engineering, Rice University \\ e-mail: \{venkatc, wakin, drorb, richb\}@rice.edu ; web: dsp.rice.edu
}

\begin{abstract}
Discontinuities in data often provide vital information, and representing these discontinuities sparsely is an important goal for approximation and compression algorithms. Little work has been done on efficient representations for higher dimensional functions containing arbitrarily smooth discontinuities. We consider the $N$-dimensional Horizon class - N-dimensional functions containing a $C^{K}$ smooth $(N-1)$-dimensional singularity separating two constant regions. We derive the optimal rate-distortion function for this class and introduce the multiscale surflet representation for sparse piecewise approximation of these functions. We propose a compression algorithm using surflets that achieves the optimal asymptotic rate-distortion performance for Horizon functions. This algorithm can be implemented using knowledge of only the $N$-dimensional function, without explicitly estimating the $(N-1)$-dimensional discontinuity.
\end{abstract}

\section{INTRODUCTION}

Discontinuities are prevalent in real-world data and often represent a boundary separating two regions, thus providing vital information. Representing discontinuities sparsely is therefore an important goal for approximation and compression algorithms. Wavelets model smooth regions well, but fail to represent discontinuities sparsely and capture their coherent nature. Little work has been done on efficient representations for higher dimensional functions with discontinuities along smooth manifolds. There are a variety of situations such as video (3-D) and light-field imaging (4-D, 5-D) for which such representations would be useful.

\section{Definitions And Problem Setup}

Let $b$ be a function of $N-1$ variables such that $b$ : $[0,1]^{N-1} \rightarrow[0,1]$. Let $\mathbf{x} \in[0,1]^{N}$ and $\mathbf{y}=\left[x_{1}, \cdots, x_{N-1}\right] \in$ $[0,1]^{N-1}$. We define the function $f$ of $N$ variables such that $f(\mathbf{x})=\mathbb{1}_{\left\{x_{N} \geq b(\mathbf{y})\right\}}$, where $\mathbb{1}_{\{\cdot\}}$ is an indicator function.

The function $f$ is known as a Horizon-class function [1] where the function $b$ defines a manifold horizon boundary between values 0 and 1 . We consider the case where the horizon $b$ belongs to $C^{K}$, where $K=r+\alpha, r$ is a positive integer, $\alpha \in(0,1]$, and all order- $r$ partial derivatives of $b$ exist and belong to $\operatorname{Lip}(\alpha)[2]$. We denote the class of all Horizon-class functions $f$ containing such a discontinuity by $\mathcal{F}$.

We have proved [2] that the optimal asymptotic ratedistortion performance for the class $\mathcal{F}$ is given by $D_{2}\left(f, \widehat{f}_{R}\right) \lesssim$ $\left(\frac{1}{R}\right)^{\frac{K}{N-1}}$, where $f \in \mathcal{F}, \widehat{f}_{R}$ is the approximation provided by the compression algorithm using $R$ bits, and $D_{2}(\cdot, \cdot)$ denotes

\footnotetext{
${ }^{1}$ This work was supported by NSF grant CCR-9973188, ONR grant N00014-02-1-0353, AFOSR grant F49620-01-1-0378, and the Texas Instruments Leadership University Program.
}

square-error distortion. The primary goal of our research is to encode an arbitrary function $f \in \mathcal{F}$ while asymptotically achieving the rate-distortion performance.

\section{SURFLETS}

A surflet at scale $j$ is an $N$-dimensional atom defined over a dyadic hypercube domain $\left[0,2^{-j}\right]^{N}$. It is a Horizon-class function with the discontinuity being a polynomial of degree $r$. One can cover the entire domain $[0,1]^{N}$ with a collection of such dyadic hypercubes (possibly at different scales) and use surflets to approximate $f$ locally over each of these smaller domains. Note that defining surflets in $N$ dimensions enables our algorithm to operate directly on the $N$-dimensional function, without explicit knowledge of the $(N-1)$-dimensional discontinuity.

We obtain a discrete surflet dictionary by quantizing the set of allowable surflet polynomial coefficients. For $\ell \in$ $\{0,1, \ldots, r\}$, a surflet coefficient of order $\ell$ at scale $j \in \mathbb{N}$ is restricted to values $\left\{n \cdot \Delta_{\ell, j}\right\}_{n \in \mathbb{Z}}$, where the stepsize satisfies $\Delta_{\ell, j}=2^{-(K-\ell) j}$. These quantization stepsizes are carefully chosen to ensure the proper fidelity of surflet approximations without requiring excess bitrate. We quantize and encode higher-order polynomial coefficients with lesser precision, without a substantial increase in distortion.

\section{Compression Algorithm}

We propose a tree-based multiresolution approach to approximate and encode $f$. The approximation is arranged on a $2^{N}$-tree, where each node in the tree at scale $j$ represents a hypercube of sidelength $2^{-j}$. Every node is either a leaf node (hypercube), or has $2^{N}$ children nodes (children hypercubes that perfectly tile the volume of the parent hypercube). Each node in the tree is labeled with a surflet. Leaf nodes provide the actual approximation to the function $f$, while interior nodes are useful for predicting and encoding their descendants. Combining the notion of multiresolution with predictive coding provides significant gains in terms of rate-distortion performance. In fact, we have proved [2] that a top-down predictive surflet coding algorithm achieves the above optimal asymptotic performance for the class $\mathcal{F}$. Finally, we note that with a minor modification, our algorithm can also achieve the optimal asymptotic rate-distortion performance when the degree of smoothness $K$ is an unknown integer.

\section{REFERENCES}

[1] D. L. Donoho, "Wedgelets: nearly-minimax estimation of edges," Annals of Stat., vol. 27, pp. 859-897, 1999.

[2] V. Chandrasekaran, M. B. Wakin, D. Baron, and R. G. Baraniuk, "Compression of higher dimensional functions containing smooth discontinuities," in 38th Ann. Conf. on Information Sciences and Systems - CISS 2004, Princeton, NJ, March 2004. 